\title{
Numerical and Experimental Study of the Structural Color by Widening the Pore Size of Nanoporous Anodic Alumina
}

\author{
Jiawen Li, Zhiqiang Zhu, Yanlei Hu, Jinjin Zheng, Jiaru Chu, and Wenhao Huang \\ Department of Precision Machinery and Precision Instrumentation, University of Science and Technology of China, \\ Hefei 230026, China \\ Correspondence should be addressed to Jiawen Li; jwl@ustc.edu.cn
}

Received 10 December 2013; Revised 8 February 2014; Accepted 10 February 2014; Published 29 April 2014

Academic Editor: Takuya Tsuzuki

Copyright (C) 2014 Jiawen Li et al. This is an open access article distributed under the Creative Commons Attribution License, which permits unrestricted use, distribution, and reproduction in any medium, provided the original work is properly cited.

\begin{abstract}
The structural color originated from the nanoporous anodic alumina (NAA) film is related to the structural characteristics. This paper aimed to obtain different structural colors which can cover the whole visible range by widening the pore size of metalcoated NAA. First, we used the Finite Difference Time Domain (FDTD) method to analyze the relationship between the physical structure and optical properties. Then, we fabricated different colors and expected color pattern by widening the pore diameter of NAA. Numerical and experimental study shows that the colors can cover the whole visible range by widening the pore diameter. This work can not only lead to better understanding of the mechanism of tuning color on NAA film, but also help us to fabricate expected color in the whole light range.
\end{abstract}

\section{Introduction}

Nanoporous anodic alumina (NAA) has stimulated considerable attention in nanoscience and engineering because it has many promising applications [1-7]. It is one of porous materials which consists of many parallel straight cylindrical nanopores perpendicular to the template surface [8]. The pore size is smaller than the wavelengths of even ultraviolet radiation and the NNA template supported on $\mathrm{Al}$ substrate can produce structural color [9].

In recent years, many researchers have focused on obtaining different structural colors on the NAA membranes by different methods such as growing nanowires [10], tuning pore depth [11], sputtering metal on NAA surface [12], and depositing carbon on the inner walls of pores [9]. The structural color on the NAA template is originated from physical structure and it is related to the structural characteristics [13]. In our previous work, we obtained some different colors on metal-coated NAA template by enlarging the pore diameter [14], but some issues had not be taken into further investigation. For example, does this method have ability to obtain colors covering the whole visible light range? How the pore feature size (pore depth, pore diameter) affects the structural color and how to get expected color?
It is generally recognized that the metal-coated NAA template is composed of metal layer, $\mathrm{Al}_{2} \mathrm{O}_{3}$ layer, barrier layer, and $\mathrm{Al}$ substrate layer [15]. The mechanisms of structural colors are often explained by Bragg's equation $[9,11]$. The peak position of reflective spectra is mainly determined by the effective refractive index and thickness of each layer of NAA template $[13,16]$. Changing the refractive index or changing layer thickness will shift the peak position [17] and leads to color changing [18]. Bragg's equation can be used to explain the mechanism of tuning color but not get desirable color for some reasons. The first is that Bragg's equation only gives peak position of reflective spectra but it cannot obtain the color because the color is calculated from reflective spectra rather than its peak position. The second is that the refractive index of each layer is varying with wavelength and it cannot be regarded as a constant value $[19,20]$. The third is that the physical model is simplified as a multilayer model. As we know, the approximate structural model of the NAA template is required in order to get accurate results and it is very important for us to design expected color. The Finite Difference Time Domain (FDTD) method can solve the problems mentioned above. It is one of the most powerful numerical techniques for studying the interaction of light with the complex structures if the structure and its dielectric 


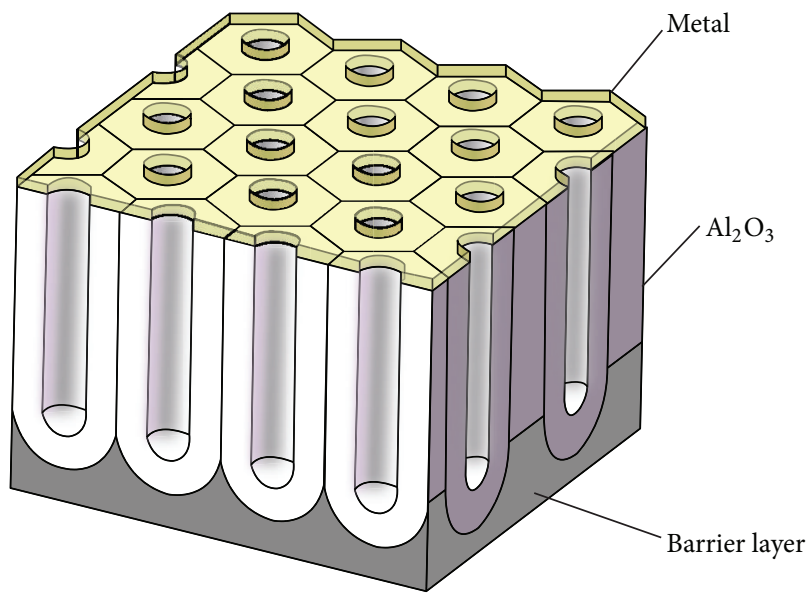

(a)

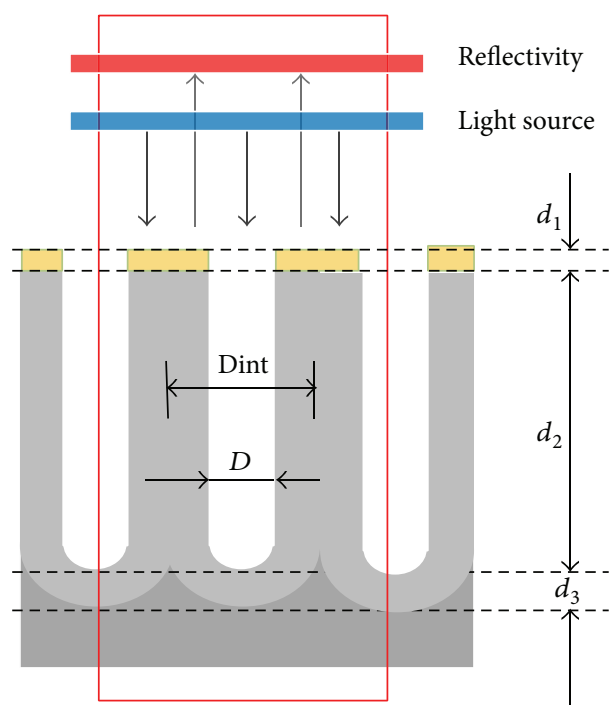

(b)

Figure 1: (a) Three-dimensional structural model of metal-coated NAA film. (b) The illustrated structure simulated by the FDTD method.

permittivity can be described [21-23]. To our knowledge, there are few papers to use FDTD method to analyze the reflective spectra of the NAA template and design expected color by changing the pore diameter.

In this paper, we aimed to obtain different structural colors which can cover the whole visible range by widening the pore parameter of NAA. First, we used FDTD method to analyze the relationship between the physical structure and optical properties. Then, we fabricated different colors by widening the pore size of NAA. Numerical and experimental study shows that the colors can cover the whole visible range using this method. This work can not only help us to fabricate expected color by widening the pore size, but also lead to better understanding of the mechanism of tuning color on NAA film.

\section{Model and Simulation}

In order to investigate the feasibility of tuning color in the whole visible range by widening pore size, we should know whether structural colors in whole visible light range can be obtained by changing pore diameter. And the processing tolerance of pore depth in widening process should be taken into consideration to get desirable color. Besides, the effect of color tuning ability should also be discussed. The structural color originated from metal-coated NAA template is related to its structural characteristics. The three-dimensional structural model of the metal-coated NAA template is illustrated in Figure 1(a). All important geometric parameters are shown in Figure 1(b). The parameter $D$ is pore diameter and Dint is inter-pore distance. The thicknesses of metal layer, $\mathrm{Al}_{2} \mathrm{O}_{3}$ layer, and barrier layer are represented by $d_{1}, d_{2}$, and $d_{3}$.

The commercial software tool FDTD Solutions is used to simulate the reflective spectra of NAA template and then the color can be calculated from the reflective spectra. The structure in simulation areas is composed of five cells, which has one cells in center and the other four are adjacent to the center with a quarter part. Periodic boundary conditions and perfectly matched layer (PML) are selected as the boundary conditions in horizontal direction and vertical direction, which is perpendicular and parallel to cylindrical nanopores, respectively. The refractive index of each layer, which shows dependence on the wavelength in the visual range, is selected from material database provided by this software. A plane wave source is located above the structure and emits a spectrally broad (360-850 nm) plane wave with a Poynting vector perpendicular to the substrate surface. The spectral reflectance is determined for each wavelength by calculating the surface integral of the real part of the Poynting vector over the detector surface and normalizing it to the incident source power. The analysis and graphical representation of the simulation data is done with Matlab software. The calculated spectrum is the average of transverse-electric (TE) and transverse-magnetic (TM) waves [24].

The optical simulated results are interpreted using colorimetric theory in terms of human color vision. We use the CIE (International Commission on Illumination) normalized illuminant D65, which closely matches that of the sky daylight. The reflective spectra are converted into tristimulus $\mathrm{XYZ}$ values and then transform the XYZ components into chromaticity coordinates xyz, which can be seen more conveniently for the CIE 1931 color space chromaticity diagram [25].

2.1. Effect of Structural Color by Changing Pore Diameter. The $\mathrm{Al}_{2} \mathrm{O}_{3}$ layer is a hexagonal porous structure and the effective refractive index is related to the filling factor of air which is determined by the pore diameter. According to Bragg's equation, the peak of reflective spectra will shift when the pore diameter is changed. We used FDTD method to simulate reflective spectra of NAA film with different pore diameter. The incident light and viewing directions were perpendicular 


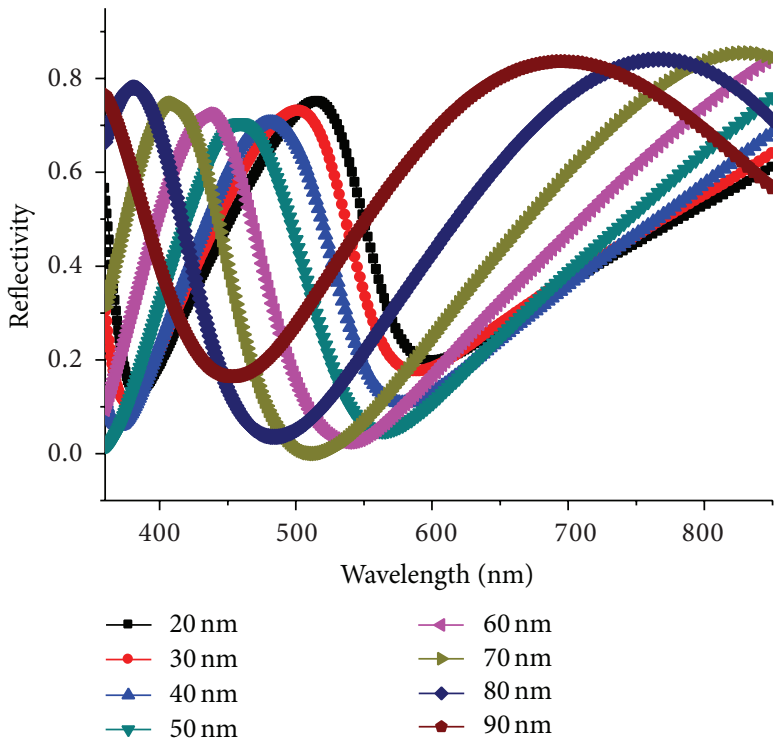

(a)

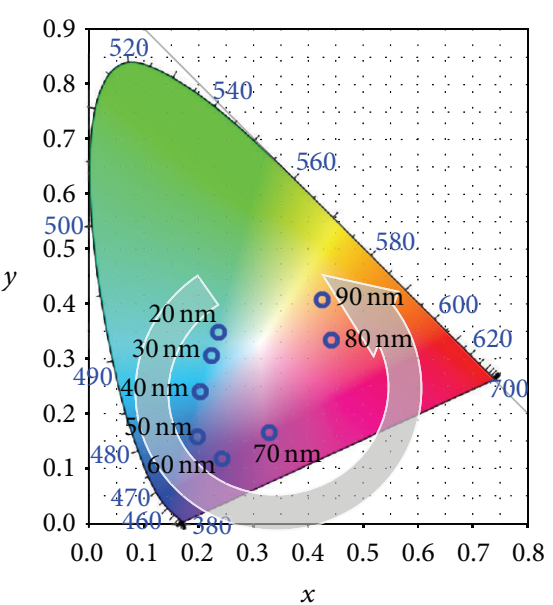

(b)

FIGURE 2: Effect of structural color by changing pore diameter: (a) reflective spectra of metal-coated NAA with different pore diameter, (b) 1931 International Commission on Illumination (CIE) chromaticity diagram with the color coordinates in (a).

to NAA surface. All the pore depths were $250 \mathrm{~nm}$. The reflective spectra of NAA with different pore diameters are shown in Figure 2(a) and the corresponding colors are shown in Figure 2(b). It can be seen that the spectra will shift when the pore diameter increases from $20 \mathrm{~nm}$ to $90 \mathrm{~nm}$. And the corresponding chromaticity coordinates in the chromaticity diagram formed an approximate circle. It can prove that the color on the metal-coated NAA can be tuned by changing the pore diameter and the color can cover almost the whole visible light range.

2.2. The Color Tuning Ability Influenced by Processing Tolerance in Widening Process. Some researchers can obtain different colors on metal-coated NAA template by changing the pore depth $[11,13]$. In this paper, we proposed a method to obtain different color by changing the pore diameter. In order to verify our method feasible, the processing tolerance to pore depth in fabrication must be taken into consideration. We used the pore widening process to enlarge the pore diameters of NAA. Because the widening process may cause the pore depth little change, we should investigate the color tuning ability influenced by processing tolerance in widening process.

It is reported that the pore widening process also thins the barrier layer on the bottom of the NAA pore array [26]. If the thinning rate of top surface in phosphoric acid solution is equal to the etched rate of the barrier layer, the pore depth has not changed. In this case, the color change is only caused by the change of the pore diameter. Otherwise, the pore depth will be changed. Because phosphoric acid etches the NAA pores isotropically in pore widening process, the top surface of NAA will also be etched [10]. If the top surface is not etched, the variation of pore depth can be considered as the etched thickness of barrier layer. If the pore diameter increases from $20 \mathrm{~nm}$ to $90 \mathrm{~nm}$ with an interval of $10 \mathrm{~nm}$, the pore depth increases with an interval of $5 \mathrm{~nm}$ which is equal to increasing pore radius size. The reflective spectra of NAA with increasing pore diameter simulated by FDTD method are shown in Figure 3(a) and the corresponding colors are shown in Figure 3(b). It can be seen that the spectra will shift when the pore diameter increases. The bright color can cover almost whole visible light range, though the intensity of corresponding color in Figure 3(b) is lower than that of Figure 2(b) and the corresponding color in Figure 3(b) is influenced by the pore depth tolerance. It can be concluded that the colors which can cover the whole visible light range can be obtained by widening pore diameter of NAA. This demonstrated that the method has color tuning ability in the whole visible range even if the pore depth has little change in the widening process.

\subsection{The Color Tuning Ability Effected by the Pore Depth. From} the Bragg equation [11], $m$ is the order number of reflective light and it is mainly determined by the pore depth. Only the peak which is distributed in the visible range will be contributed to the observed color. The observed color is a mixture of multicolors corresponding to wave peaks. The redshift of the peak will occur in every corresponding $m$ with pore depth decreasing and redshift will skip when the value of $m$ changes. When the pore depth is $150 \mathrm{~nm}$, the reflective spectra in Figure 4(a) with different pore diameters only have one peak and $m=1$, which stands for the monochromaticity on the samples. With the pore diameter increasing, the color has little change and color saturation is very low, seen from Figure 4(b). In this case, the color tuning ability is very bad. When the pore depth is $300 \mathrm{~nm}$, the reflective spectra in 


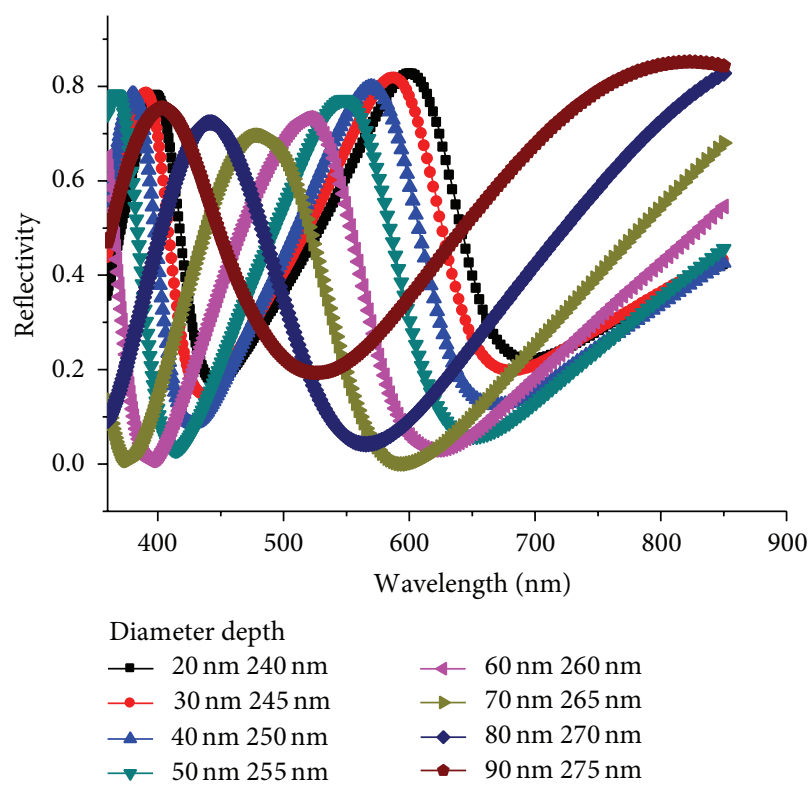

(a)

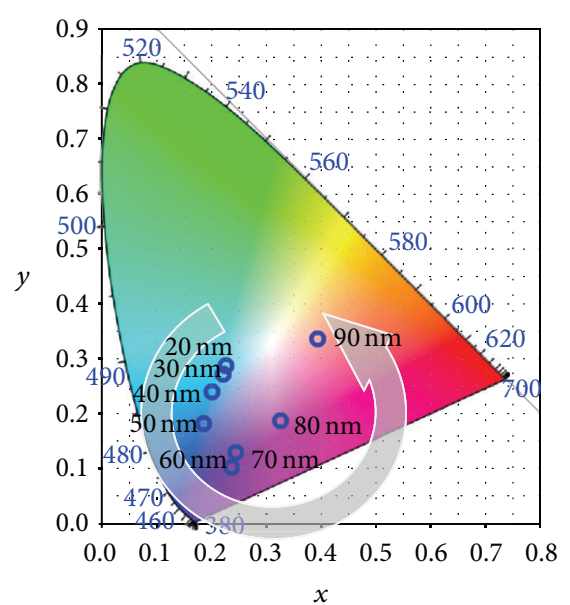

(b)

FIGURE 3: Color tuning ability caused by processing tolerance: (a) reflective spectra of NAA with different pore diameter and different pore depth, (b) 1931 International Commission on Illumination (CIE) chromaticity diagram with the color coordinates in (a).

Figure 4(c) with different pore diameters have two peaks and $m=2$. The color will dramatically change when the pore diameter increases, shown in Figure 4(d). The colors can cover the whole visible range and have high saturation. It can be seen that the color tuning ability will be affected by the pore depth. So an appropriate depth should be selected in order to get long color tuning range by changing the pore diameter.

\section{Experimental Section}

The structural characteristics of the NAA such as pore diameter, pore depth, interpore distance, and barrier layer thickness can be tuned by simple variation of the anodization parameters, such as anodizing voltage and electrolyte solution composition. In this paper, two-step anodization procedure was carried to prepare the NAA membrane and widening process was used to enlarge pore diameter.

High-purity Al foils (99.999\%) with $250 \mu \mathrm{m}$ thickness were first ultrasonic cleaned in acetone, ethanol, and distilled water. Subsequently, the $\mathrm{Al}$ foils were annealed at $500^{\circ} \mathrm{C}$ under vacuum ambient for 5 hours to remove the mechanical stress, and surface roughness was reduced by an electrochemically polished process by a mixture of $\mathrm{HClO}_{4}$ and $\mathrm{C}_{2} \mathrm{H}_{5} \mathrm{OH}$ (volume ratio 1:4). In the first anodization step, the aluminum foils were anodized in oxalic acid solution under a constant voltage of $40 \mathrm{~V}$ at $5^{\circ} \mathrm{C}$ for 2 hours. Then the oxide layer on the surface was removed by immersing the foils in a mixture of $\mathrm{H}_{3} \mathrm{PO}_{4}(6.0 \mathrm{wt} \%)$ and $\mathrm{CrO}_{3}(1.8 \mathrm{wt} \%)$ at $60^{\circ} \mathrm{C}$ for 4 hours. In the second anodization, the pretreated aluminum foils were performed using the same anodized conditions as at the first step except for anodization times which is related to the pore depth. After the two-step anodization procedure, the prepared the NAA membranes were immersed into $\mathrm{H}_{3} \mathrm{PO}_{4}$ (6.0wt\%) to enlarge pore diameter.

A Field Emission Scanning Electron Microscopy (FESEM) (JSM-6700F) is used to characterize NAA template. The cross-sectional and top-view images in Figure 5 show that this preparation method can get well-aligned and high quality NAA film. It can be seen that, during the pore widening process, the pore diameter enlarged while interpore distance remained fixed. The average interpore distance is about $100 \mathrm{~nm}$. The relationship between the pore diameter and pore widening process time can be seen in Figure 5(e). The pore diameter of the film before pore widening process is about $37 \mathrm{~nm}$. With the pore widening time increasing, the pore diameter of NAA is increased. The linear curve can be achieved by plotting the pore diameter to pore widening time with $y(\mathrm{~nm})=34.94+0.64 x(\mathrm{~min})$. It is concluded that different widening process times could be used to obtain NAA film with different pore diameter.

\section{Result and Discussion}

To verify the feasibility of this method, 11 NAA samples with various pore diameters were prepared and then sputtered with a Pt nanolayer with a thickness of about $7.5 \mathrm{~nm}$. As a result, a series of brilliant colors are exhibited, such as yellow, orange, red, purple, blue, and green, which can be seen in Table 1. The optical reflective spectra of these NAA films are characterized using laser scanning confocal microscope Raman spectrometer in which light source is replaced by visible-NIR light source and $\mathrm{BaSO}_{4}$ is used as a reference. The reflective spectra and the color hues of these samples and are 


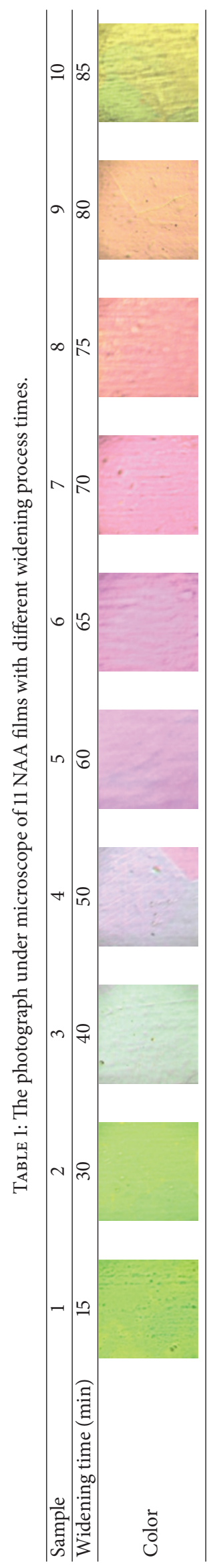




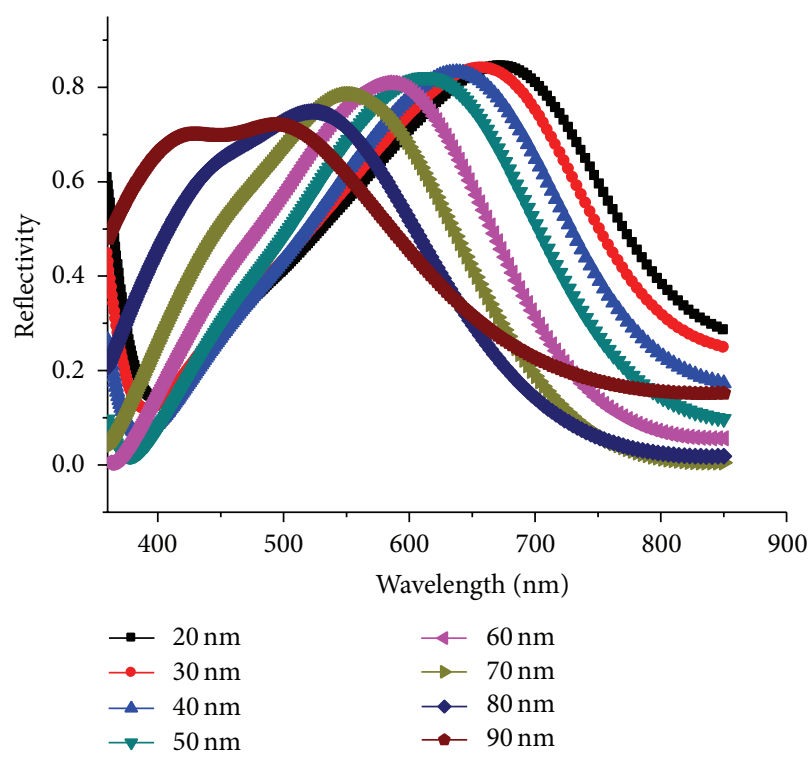

(a)

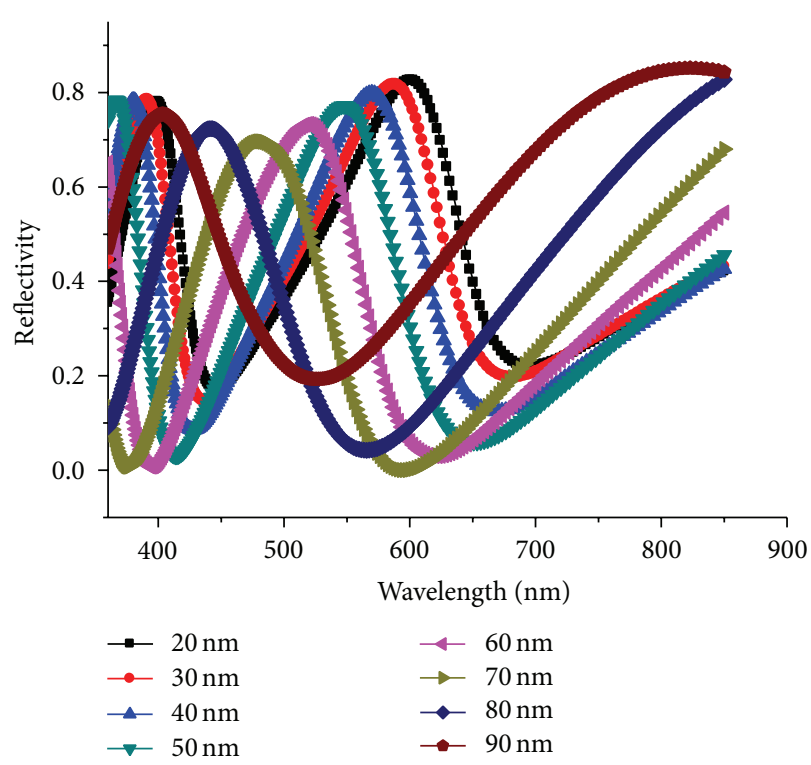

(c)

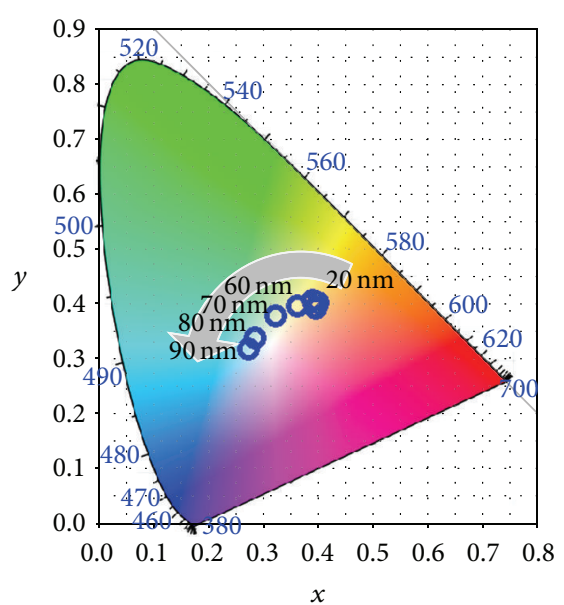

(b)

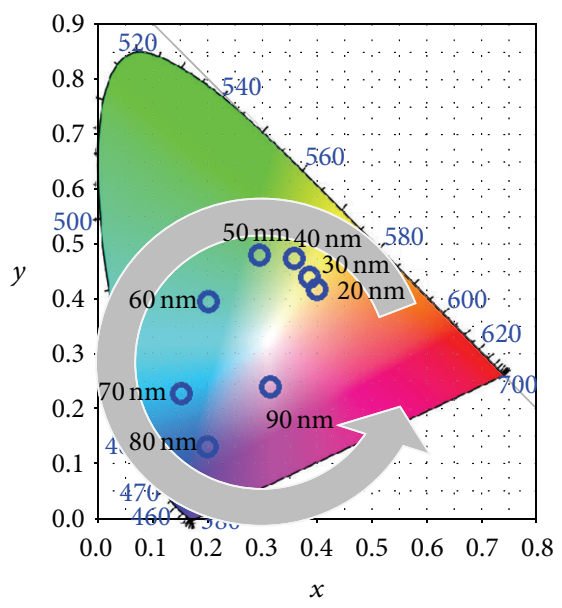

(d)

FIGURE 4: Effect of structural color caused by pore depth: (a) reflective spectra of NAA with different pore diameter and $150 \mathrm{~nm}$ pore depth, (b) 1931 International Commission on Illumination (CIE) chromaticity diagram with the color coordinates in (a), (c) reflective spectra of NAA with different pore diameter and $300 \mathrm{~nm}$ pore depth, and (d) 1931 International Commission on Illumination (CIE) chromaticity diagram with the color coordinates in (c).

shown in Figure 6. The peak position of the reflective spectra in each curve changed with widening process times. When the widen process time was increased, the peak position will shift. It is an interesting finding that a circulation of color variation is formed and the color can cover almost the whole visible light range. As we know, the color is not only related to the pore diameter but also related to the pore depth. Pore depth is determined by the anodization time in the two-step anodization process. In our experiment, we wanted to fabricate the samples in which the pore depths were about $250 \mathrm{~nm}$ first. But we could not control the pore depths accurately. We used the ImageJ software to calculate the pore depths from the cross-section SEMs. We found that the depths were little bigger than that we expected. The depths were about $270 \mathrm{~nm}$. We think the thinning rate of top surface in phosphoric acid solution is almost equal to the etched rate of the barrier layer [10]. Each color was located at the corresponding coordinate between Figure 2(b) and Figure 4(d). So it can be seen that all kinds of colors can be successfully obtained by tuning the pore diameters and the color can cover almost visible range. This means we can get expected color by widening pore diameter method. 


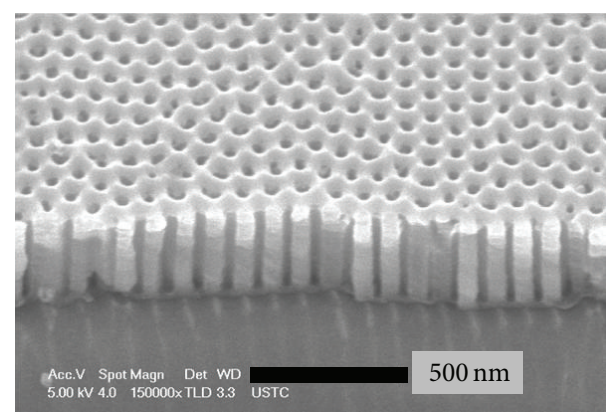

(a)

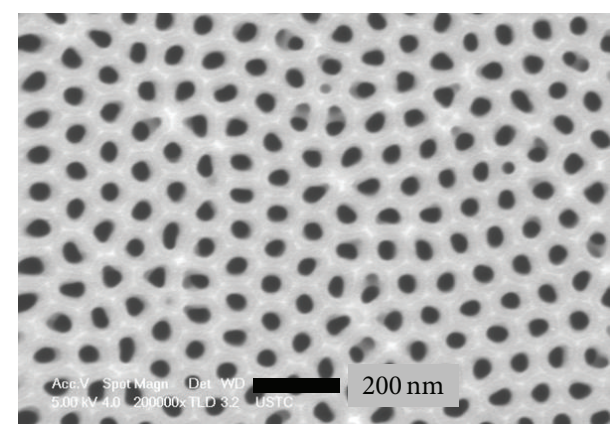

(c)

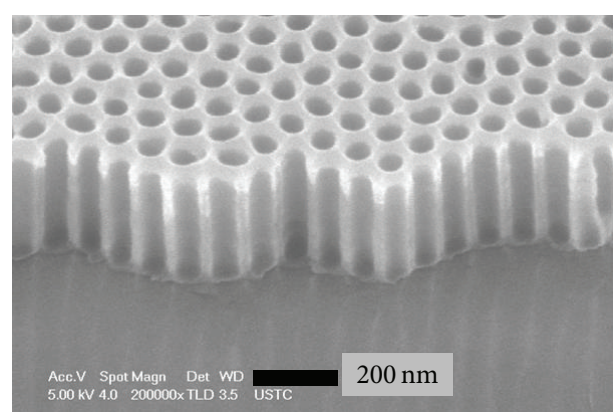

(b)

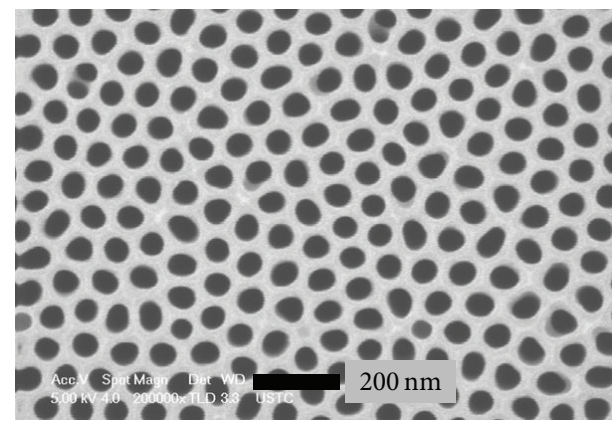

(d)

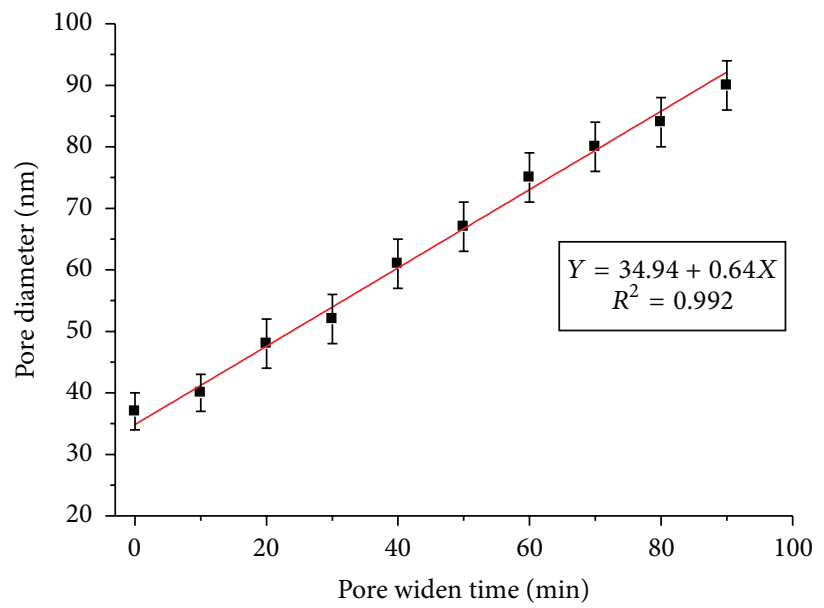

(e)

FIGURE 5: Effect of pore widening time on the pore sizes: (a) cross-sectional image of NAA film before the pore widening process, (b) crosssectional image of NAA film with pore widening for $30 \mathrm{~min}$, (c) top-view image of NAA films before the pore widening process, (d) top-view image of NAA films with pore widening for $30 \mathrm{~min}$, (e) and the variation of the pore diameter as a function of the pore widening time.

In order to produce a colorful pattern on a film using method, we fabricated the NAA template with different pore diameter in different areas. The photoresist was used as mask to obtain two pore diameters on the same NAA film and the process is illustrated in Figure 7. Firstly, the photoresist covered on the film was exposed through a pattern of intense light by an exposure system (model: ABM/6/350). The unexposed photoresist was removed by wash fixer solution and the exposed photoresist remained on the film surface. Then, the NAA sample was immersed in $\mathrm{H}_{3} \mathrm{PO}_{4}$ (6 wt\%) solution for some time and the pores of NAA on unexposed area were enlarged. Finally, the rest of photoresist on NAA film was removed. To get color with high saturation, Pt metal was sputtered on the prepared NAA template with $7.5 \mathrm{~nm}$ thickness.

Using this method, an expected colorful pattern exhibited on the NAA surface under daylight was successfully fabricated as presented in Figure 8. The pore diameters of pattern substrates had not been widened because of covered photoresist. And pore diameters of letter pattern ("USTC") have been widened and the color is related to the widening process time. It can be seen that the colors of widened part are different from that of unwidened part. If the concentration of acid solutions, anodic voltage, and the temperature of 


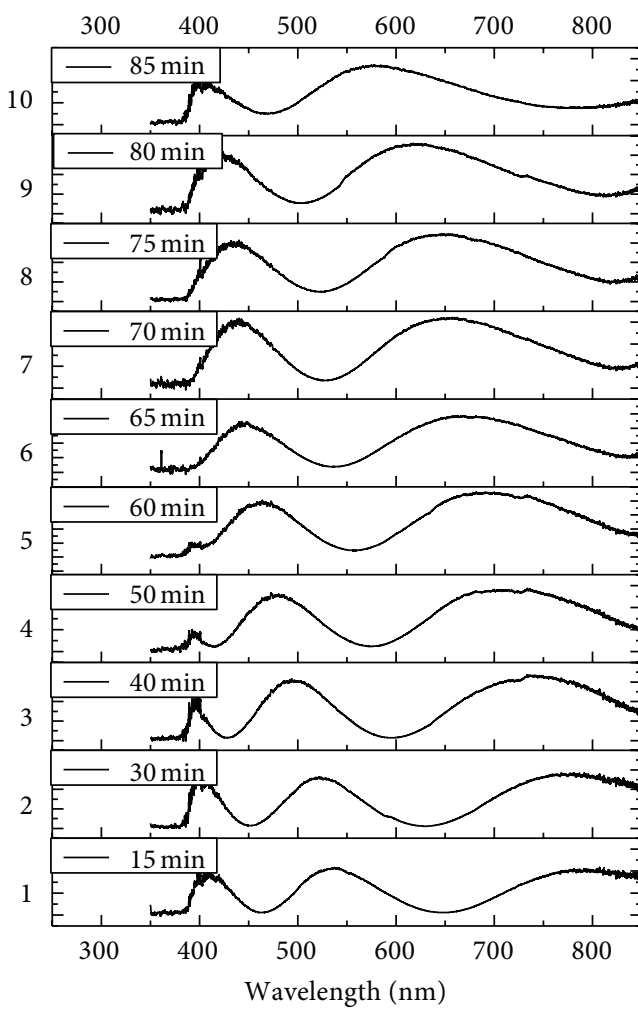

(a)

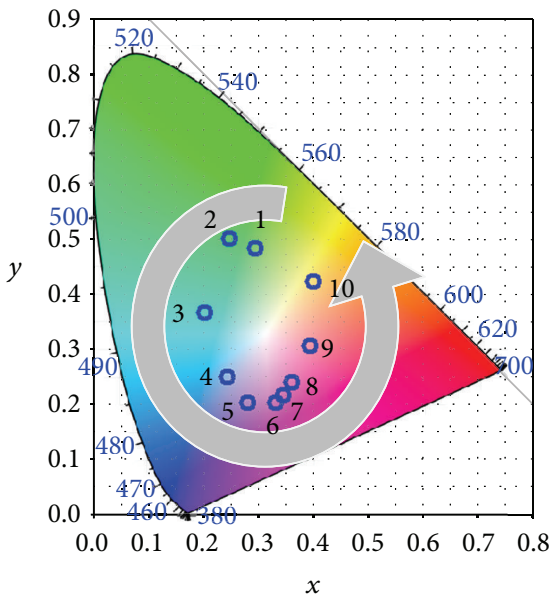

(b)

FIGURE 6: (a) Reflective spectra of NAA films with different widening process times measured by spectrometer, (b) 1931 International Commission on Illumination (CIE) chromaticity diagram of the NAA films with different widening process times.

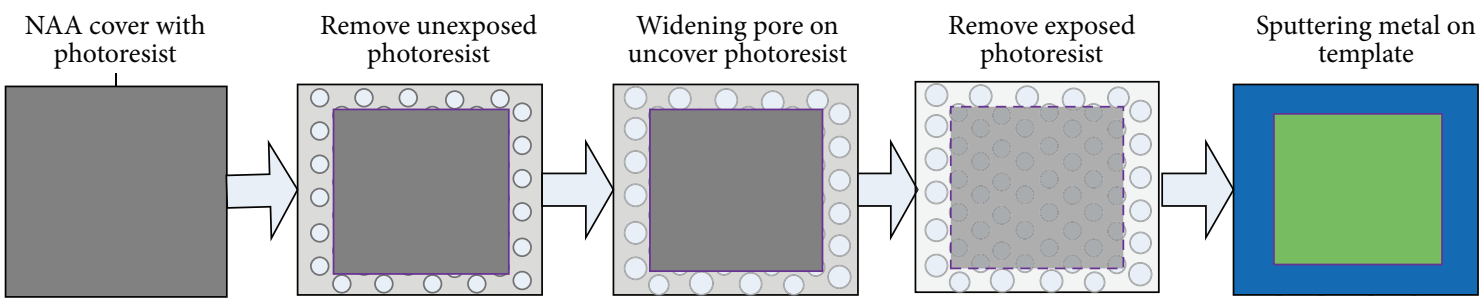

FIGURE 7: The flowchart to interpret the preparation of the complex color pattern.

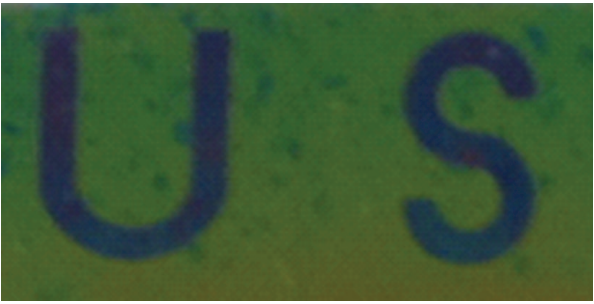

(a)

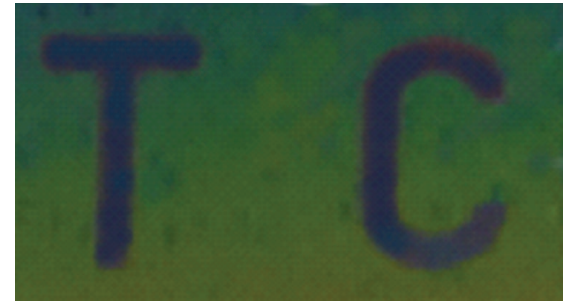

(b)

FIGURE 8: Colorful patterns prepared on Pt-coated NAA template with two pore diameters. It can be inferred that different color can be obtained by tuning pore diameter in different areas on NAA film surface. 
electrolyte can controlled well, all the letters will have uniform color. The result verified the feasibility and practicality of this method for color tuning on NAA templates.

\section{Conclusion}

We developed a fascinating method to obtain different structural colors which can cover the whole visible range by changing the pore diameter of NAA. First, we used Finite Difference Time Domain (FDTD) method to analyze the relationship between the structural color and the parameters of physical structure. The different colors covering the whole visible light range can be acquired by changing the pore diameter. Then, we prepared the NAA membrane by twostep anodization procedure and then immerse the fabricated NAA membrane into phosphoric acid to enlarge the pore diameter. The different pore diameters of NAA were obtained. By sputtering metal on surface of NAA, different colors of NAA film covering almost the whole visible light range were obtained and colorful patterns were successfully fabricated. This work can not only lead to better understanding the mechanism of tuning color on NAA film, but also help us to fabricate desirable color. This method can be used in display, decoration, anticounterfeiting, and environmental sensors.

\section{Conflict of Interests}

The authors declare that there is no conflict of interests regarding the publication of this paper.

\section{Acknowledgments}

This work is supported by National Science Foundation of China (nos. 51275502, 51205375), Anhui Provincial Natural Science Foundation (no.1408085ME104), National Basic Research Program of China (no. 2011CB302100), China Postdoctoral Science Foundation Funded Project (nos. 2012M511416, 2012M521245), and the Fundamental Research Funds for the Central Universities (KB2090090001).

\section{References}

[1] D. Bruggemann, "Nanoporous aluminium oxide membranes as cell interfaces," Journal of Nanomaterials, vol. 2013, Article ID 460870, 18 pages, 2013.

[2] C.-H. Huang, H.-Y. Lin, Y. Tzeng et al., "Optical characteristics of pore size on porous anodic aluminium oxide films with embedded silver nanoparticles," Sensors and Actuators A: Physical, vol. 180, pp. 49-54, 2012.

[3] W. Zhou, X. Niu, G. Min et al., "Porous alumina nano-membranes: soft replica molding for large area UV-nanoimprint lithography," Microelectronic Engineering, vol. 86, no. 12, pp. 2375-2380, 2009.

[4] C. Song, W. Shi, H. Jiang, J. Tu, and D. Ge, "PH-sensitive characteristics of poly(acrylic acid)-functionalized anodic aluminum oxide (AAO) membranes," Journal of Membrane Science, vol. 372, no. 1-2, pp. 340-345, 2011.

[5] Z. Jin, F. Meng, J. Liu, M. Li, L. Kong, and J. Liu, "A novel porous anodic alumina based capacitive sensor towards trace detection of PCBs," Sensors and Actuators B: Chemical, vol. 157, no. 2, pp. 641-646, 2011.

[6] B. Wang, G. T. Fei, M. Wang, M. G. Kong, and L. D. Zhang, "Preparation of photonic crystals made of air pores in anodic alumina," Nanotechnology, vol. 18, no. 36, Article ID 365601, 2007.

[7] M. T. Rahman, H. Wang, and J.-P. Wang, "Exploration of the direct use of anodized alumina as a mold for nanoimprint lithography to fabricate magnetic nanostructure over large area," Journal of Nanotechnology, vol. 2011, Article ID 961630, 5 pages, 2011.

[8] W. Chen, J.-S. Wu, and X.-H. Xia, "Porous anodic alumina with continuously manipulated pore/cell size," ACS Nano, vol. 2, no. 5, pp. 959-965, 2008.

[9] X. H. Wang, T. Akahane, H. Orikasa, T. Kyotani, and Y. Y. $\mathrm{Fu}$, "Brilliant and tunable color of carbon-coated thin anodic aluminum oxide films," Applied Physics Letters, vol. 91, no. 1, Article ID 011908, 2007.

[10] X. Zhao, G. Meng, Q. Xu, F. Han, and Q. Huang, "Color finetuning of CNTs@AAO composite thin films via lsotropically etching porous AAO before CNT growth and color modification by water infusion," Advanced Materials, vol. 22, no. 24, pp. 2637-2641, 2010.

[11] X. Wang, D. Zhang, H. Zhang, Y. Ma, and J. Z. Jiang, “Tuning color by pore depth of metal-coated porous alumina," Nanotechnology, vol. 22, no. 30, Article ID 305306, 2011.

[12] X. Wang, H. Zhang, D. Zhang, Y. Ma, H.-J. Fecht, and J. Z. Jiang, "Color tuning by local sputtering metal nanolayer on microstructured porous alumina," Microscopy Research and Technique, vol. 75, no. 5, pp. 698-701, 2012.

[13] W. L. Xu, H. Chen, M. J. Zheng, G. Q. Ding, and W. Z. Shen, "Optical transmission spectra of ordered porous alumina membranes with different thicknesses and porosities," Optical Materials, vol. 28, no. 10, pp. 1160-1165, 2006.

[14] J. Li, Z. Zhu, P. Deng, Y. Hu, J. Chu, and W. Huang, "Brilliant and tunable color by changing pore diameter of metal-coated porous anodic alumina," in Nanophotonics and Micro/Nano Optics, Proceedings of SPIE, The Society of Photo-Optical Instrumentation Engineers (SPIE), Chinese Optical Society (COS), Beijing, China, November 2012.

[15] Q. Xu, H.-Y. Sun, Y.-H. Yang, L.-H. Liu, and Z.-Y. Li, "Optical properties and color generation mechanism of porous anodic alumina films," Applied Surface Science, vol. 258, no. 5, pp. 18261830, 2011.

[16] W. J. Zheng, G. T. Fei, B. Wang, and L. De Zhang, "Modulation of transmission spectra of anodized alumina membrane distributed bragg reflector by controlling anodization temperature," Nanoscale Research Letters, vol. 4, no. 7, pp. 665-667, 2009.

[17] B. Wang, X. P. Zhang, W. J. Song, P. Cui, Y. Zhang, and G. T. Fei, "Fabrication of transmission phase gratings on porous anodic alumina," Optics Letters, vol. 35, no. 5, pp. 727-729, 2010.

[18] Q. Xu, Y. Yang, J. Gu, Z. Li, and H. Sun, "Influence of Al substrate on the optical properties of porous anodic alumina films," Materials Letters, vol. 74, pp. 137-139, 2012.

[19] J. Chen, B. Wang, Y. Yang, Y. Shi, G. Xu, and P. Cui, "Porous anodic alumina with low refractive index for broadband graded-index antireflection coatings," Applied Optics, vol. 51, no. 28, pp. 6839-6843, 2012.

[20] J. Wang, C.-W. Wang, Y. Li, and W.-M. Liu, "Optical constants of anodic aluminum oxide films formed in oxalic acid solution," Thin Solid Films, vol. 516, no. 21, pp. 7689-7694, 2008. 
[21] M. A. Steindorfer, V. Schmidt, M. Belegratis, B. Stadlober, and J. R. Krenn, "Detailed simulation of structural color generation inspired by the Morpho butterfly," Optics Express, vol. 20, no. 19, pp. 21485-21494, 2012.

[22] J. Niu, Y. Jun Shin, Y. Lee, J.-H. Ahn, and H. Yang, "Graphene induced tunability of the surface plasmon resonance," Applied Physics Letters, vol. 100, no. 6, Article ID 061116, 2012.

[23] J. Li, Q. Han, Y. Chen, Z. Zhu, and W. Huang, "Investigation on structural colours in wing scales of butterfly Papilio peranthus Fabricius," IET Micro \& Nano Letters, vol. 8, no. 1, pp. 8-10, 2013.

[24] J. Li, Q. Han, Y. Chen, J. Chu, and W. Huang, "Tunable structural colour on the basis of colloidal crystal," Micro and Nano Letters, vol. 6, no. 7, pp. 530-533, 2011.

[25] B. Gralak, G. Tayeb, and S. Enoch, "Morpho butterflies wings color modeled with lamellar grating theory," Optics Express, vol. 9, no. 11, pp. 567-578, 2001.

[26] J. Zhang, J. E. Kielbasa, and D. L. Carroll, "Controllable fabrication of porous alumina templates for nanostructures synthesis," Materials Chemistry and Physics, vol. 122, no. 1, pp. 295-300, 2010. 

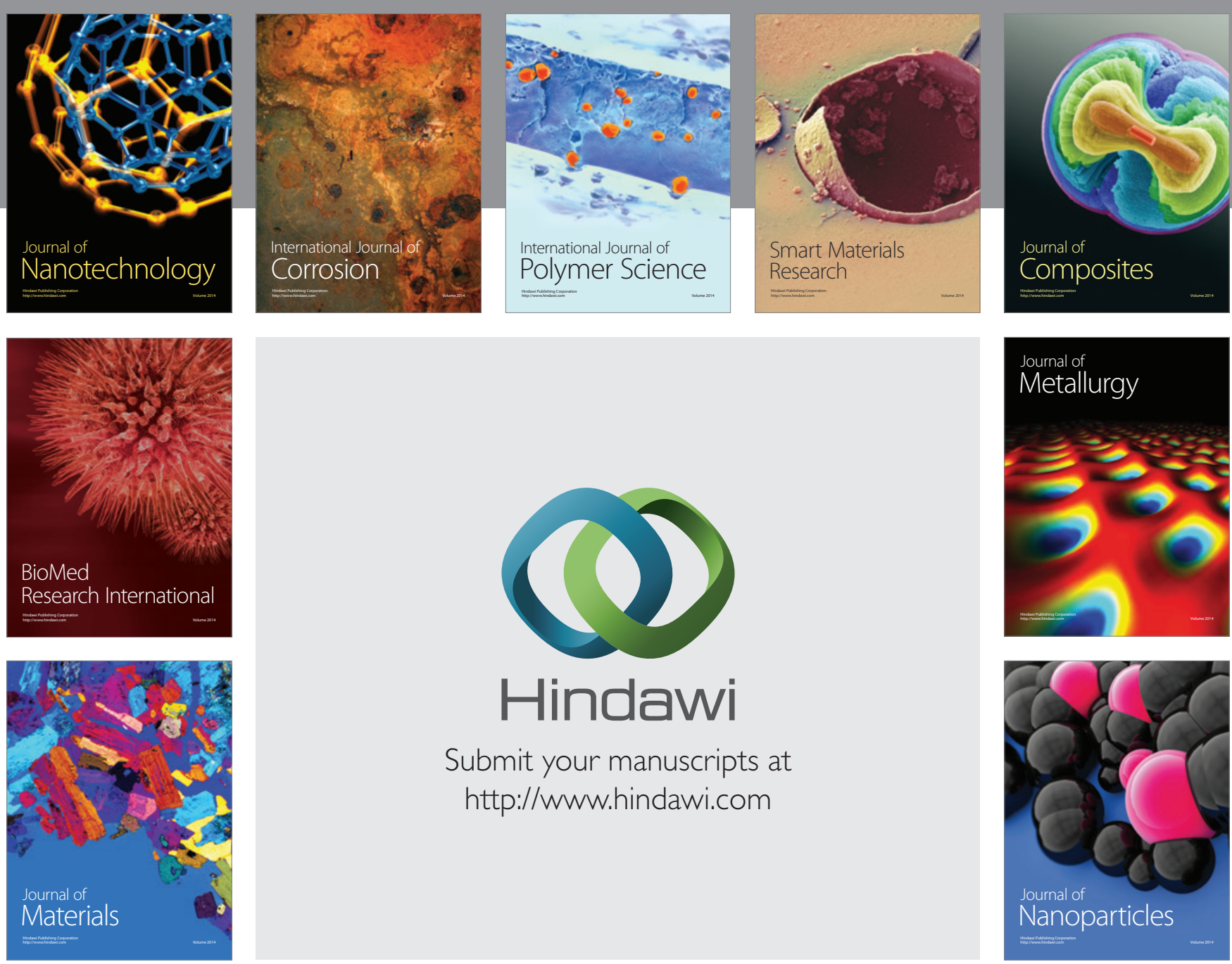

Submit your manuscripts at http://www.hindawi.com
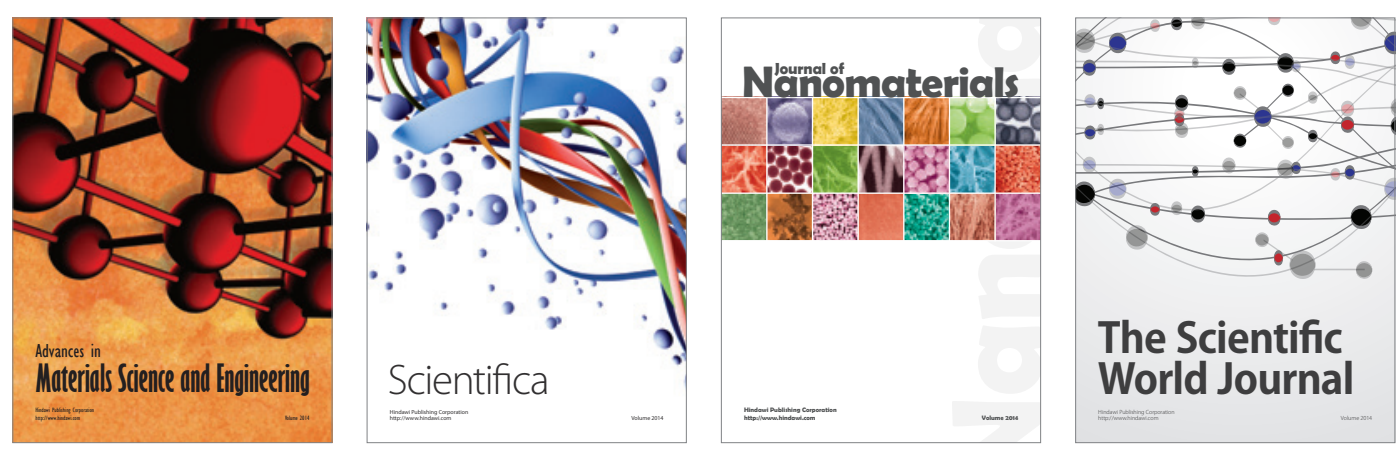

\section{The Scientific World Journal}
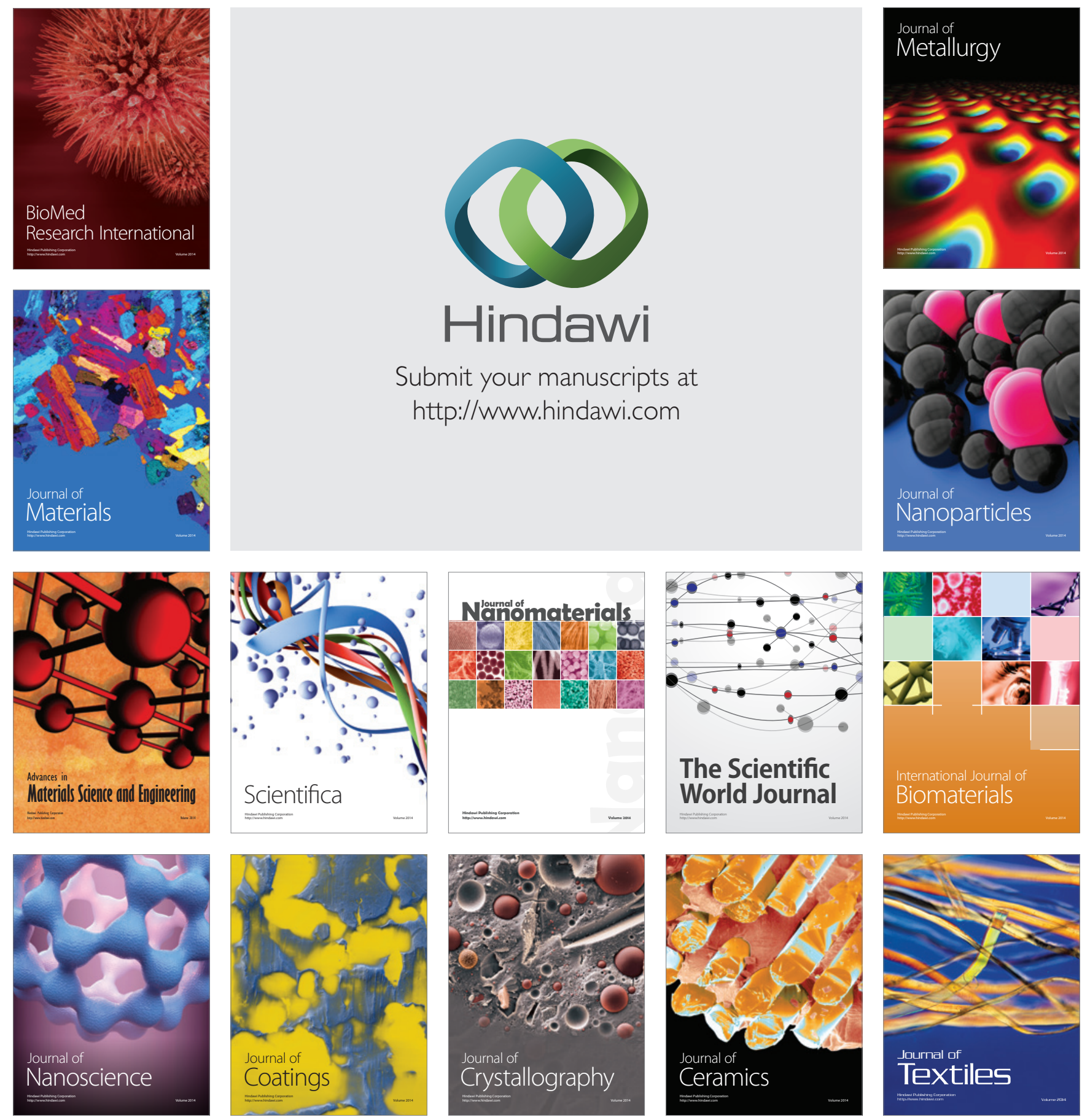